\title{
Genetic organization of pre-CTX and CTX prophages in the genome of an environmental Vibrio cholerae non-O1, non-O139 strain
}

Correspondence
Rupak K. Bhadra
rupakbhadra@iicb.res.in

Received 14 July 2006

Revised 30 August 2006

Accepted 1 September 2006
Diganta Maiti, ${ }^{1}$ Bhabatosh Das, ${ }^{1}$ Arjun Saha, ${ }^{1} \dagger$ Ranjan K. Nandy, ${ }^{2}$ G. Balakrish Nair ${ }^{3}$ and Rupak K. Bhadra ${ }^{1}$

\author{
${ }^{1}$ Infectious Diseases Group, Indian Institute of Chemical Biology, 4, Raja S. C. Mullick Road, \\ Kolkata 700 032, India \\ ${ }^{2}$ National Institute of Cholera and Enteric Diseases, Beliaghata, Kolkata 700 010, India \\ ${ }^{3}$ International Centre for Diarrhoeal Disease Research, Bangladesh, Dhaka 1212, Bangladesh
}

The cholera toxin (CT) is a critical determinant of the virulence of epidemic Vibrio cholerae strains. The ct $x A B$ operon encoding CT is part of the genome of a filamentous bacteriophage CTXФ, which may integrate as a single copy or as multiple copies in the genome of $V$. cholerae. The CTXФ genome is composed of RS2 $(2.4 \mathrm{~kb})$ and core $(4.5 \mathrm{~kb})$ regions. In the present study extensive genetic mapping analyses indicated that two copies of tandemly arrayed CTX prophages are integrated in the small chromosome of an environmental $V$. cholerae strain, VCE232, belonging to serogroup O4. Further mapping revealed that the integration of prophages has occurred in the same genetic locus of the small chromosome of VCE232 as that of V. cholerae O1 biotype El Tor strains. Interestingly, a new type of RS2-like element $3.5 \mathrm{~kb}$ in size was found in the CTX prophage genome in the small chromosome of VCE232. Cloning followed by sequencing of the new RS2-like element of VCE232 revealed the presence of three ORFs, which probably encode highly divergent types of phage regulatory proteins. Furthermore, the strain VCE232 also harbours two copies of a tandemly arranged CTX prophage devoid of the ct $A B$ genes, called pre-CTX prophage, in its large chromosome. The presence of multiple copies of diverse CTX prophages in both the chromosomes of VCE232 suggests that toxigenic environmental $V$. cholerae non-O1, non-O139 strains could play a role in the emergence of new epidemic clones.

\section{INTRODUCTION}

The aquatic environment is the natural habitat of Vibrio cholerae, which includes both pathogenic and nonpathogenic strains. However, of the more than 206 recognized 'O' serogroups of $V$. cholerae (Li et al., 2002), only the strains belonging to serogroups $\mathrm{O} 1$ and $\mathrm{O} 139$ are responsible for epidemic and pandemic cholera of humans. The other serogroups of $V$. cholerae, collectively called non-O1, nonO139, have not been associated with epidemics, but can cause sporadic diarrhoea and are ubiquitously distributed in the aquatic environment. The strains of $\mathrm{O} 1$ serogroup are further differentiated into two biotypes, classical and El Tor, on the basis of several phenotypic tests (Kaper et al., 1995).

tPresent address: Department of Developmental Genetics, NICHD, National Institutes of Health, Bethesda, MD 20892, USA.

Abbreviations: CT, cholera toxin; NCBI, National Center for Biotechnology Information.

The GenBank/EMBL/DDBJ accession number for the RS2-like sequence of $V$. cholerae strain VCE232 reported in this paper is Da288668.
The classical biotype, the sixth pandemic strain, was responsible for cholera epidemics until 1961, when the El Tor biotype displaced it and started the seventh pandemic, which is still continuing (Kaper et al., 1995). In 1992, a newly recognized serogroup, O139, emerged, and resulted in massive cholera epidemics in the Indian subcontinent (Ramamurthy et al., 1993). Several lines of evidence have suggested that strain O139 arose from an El Tor biotype by acquiring the $\mathrm{O} 139$ biosynthesis gene cluster from a non-O1 strain (Bhadra et al., 1995; Bik et al., 1995; Waldor \& Mekalanos, 1994). Thus, O139 combines the virulent properties of $\mathrm{O} 1$ strains and the presence of a capsule of a non-O1 strain.

The strains belonging to $\mathrm{O} 1$ and $\mathrm{O} 139$ produce a potent enterotoxin, called cholera toxin (CT), encoded by $c t x A B$ genes, which is influenced and regulated by environmental cues, and is central to the disease process. An important discovery in cholera research is that the $\operatorname{ct} A B$ locus is a part of the genome of a bacteriophage, called СТХ $\Phi$ (Waldor \& Mekalanos, 1996), and thus it is highly probable that this virulence locus may be transferred among diverse $V$. cholerae 


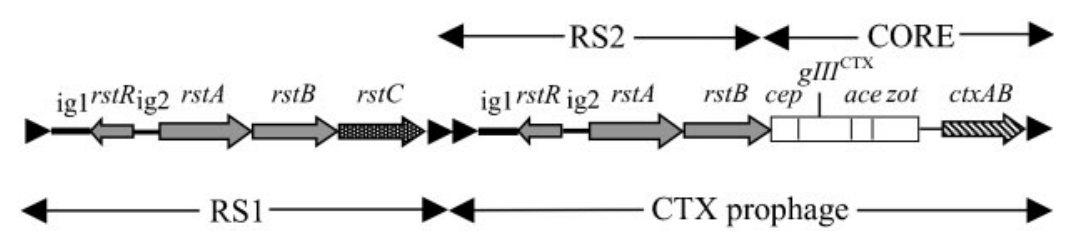

Fig. 1. Schematic representation of the canonical genetic organization of an RS1 element and a CTX prophage comprising an RS2 segment and a core region (not drawn to scale). Solid triangles represent end repeats. Block arrows represent the indicated genes present in the CTX element and their direction of transcription. strains in the environment. СТХ $\Phi$ is a single-stranded filamentous bacteriophage with a small genome of $\sim 6.9 \mathrm{~kb}$ (Waldor \& Mekalanos, 1996), and is composed of two parts, phage regulatory genetic element RS2, of $\sim 2 \cdot 4 \mathrm{~kb}$, and a core region of $\sim 4.5 \mathrm{~kb}$ (Fig. 1). The RS2 element contains three genes $5^{\prime}$-rstR-rstA-rstB-3' (Waldor et al., 1997), while the core region harbours six genes, including the $\operatorname{ct} x A B$ operon. The genes of the RS2 element encode functions required for regulation ( $r s t R$ gene product), replication ( $r s t A$ gene product) and integration ( $r s t B$ gene product) of СТХ $\Phi$ in the genome of $V$. cholerae (Waldor et al., 1997). Several distinct CTX $\Phi$ variants have been described, namely $\mathrm{CTX}^{\mathrm{ET}} \Phi$ derived from $V$. cholerae $\mathrm{O} 1 \mathrm{El}$ Tor, $\mathrm{CTX}^{\text {class }} \Phi$ derived from classical $V$. cholerae, $\mathrm{CTX}^{\mathrm{Cal}} \Phi$ derived from O139 V. cholerae (Davis et al., 1999), $\mathrm{CTX}^{\mathrm{Env}} \Phi$ derived from non-O1, non-O139 V. cholerae (Mukhopadhyay et al., $2001)$, and $\mathrm{CTX}^{\mathrm{var}} \Phi$ present in pre-O139 V. cholerae El Tor isolates (Nandi et al., 2003). Apart from these genes, some RS2 elements may contain an additional gene, $r s t C$ (Fig. 1); in this instance, the element is termed RS1 and is $2.7 \mathrm{~kb}$ in size (Waldor et al., 1997). RstC is an antirepressor that controls CTXФ lysogeny (Davis et al., 2002). RS1 generally flanks $5^{\prime}$ and/or $3^{\prime}$ of the CTX prophage genome in toxigenic $V$. cholerae $\mathrm{O} 1 \mathrm{El}$ Tor biotype and O139 strains (Davis \& Waldor, 2000), but is absent from the classical strains (Davis et al., 2000). V. cholerae possesses two circular chromosomes, one small and one large (Heidelberg et al., 2000; Trucksis et al., 1998). In V. cholerae El Tor/O139 genomes the CTX prophage may be present either as a single copy or as multiple copies arranged in tandem (Bhadra et al., 1995; Mekalanos, 1983; Nandi et al., 2003). In sharp contrast, in $V$. cholerae classical strains, the prophage is usually present in two copies, one copy in each chromosome (Nandi et al., 2003; Trucksis et al., 1998). The whole-genome sequencing of the $V$. cholerae El Tor strain N16961 has revealed the integration of only one copy of the CTX prophage flanked by the RS1 element in the large chromosome (Heidelberg et al., 2000). Recently, the integration of the tandem copies of СТXФ in the small chromosomes of pre-O139 V. cholerae El Tor strains has also been demonstrated (Nandi et al., 2003).

Unlike epidemic $V$. cholerae strains, no information is currently available about the organization of the CTX prophage and its chromosomal location in the genomes of CT-positive non-O1, non-O139 strains of $V$. cholerae. The results of this study show for the first time the presence of tandemly arrayed copies of CTX prophages in the small chromosome of a $V$. cholerae non-O1, non-O139 strain, VCE232 (serogroup O4), which was isolated from a yearlong environmental study conducted in Calcutta in the 1980s (Nair et al., 1988). Furthermore, we also mapped the large chromosomal tandem integration of two CTX prophages, both devoid of the $\operatorname{ctx} A B$ genes. Interestingly, the small chromosomal CTX prophage appears to be unique, since its genome contains a novel RS2-like element of $3.5 \mathrm{~kb}$, which was cloned and sequenced.

\section{METHODS}

Bacterial strains and growth conditions. Details of $V$. cholerae strains used in this study are described in Table 1. For cloning purposes, Escherichia coli DH5 $\alpha$ (Saha et al., 2004) was used. Bacterial strains were routinely grown either in Luria broth (LB) with shaking at $37^{\circ} \mathrm{C}$ or in $\mathrm{LB}$ containing $1.5 \%$ agar (LA) in plates, as described previously (Nandi et al., 2003). The antibiotic ampicillin was used at $100 \mu \mathrm{g} \mathrm{ml}^{-1}$ where needed. Bacterial strains were maintained at $-70{ }^{\circ} \mathrm{C}$ in LB containing $15 \%$ (v/v) glycerol (Nandi et al., 2003).

PFGE. Intact genomic DNA of $V$. cholerae was prepared and digested with the rare-cutter restriction enzyme Not (New England Biolabs), essentially as described by Nandi et al. (2003). For the

Table 1. V. cholerae strains used in this study

\begin{tabular}{|lclll|}
\hline Strain & Serotype (biotype) & \multicolumn{1}{c|}{ Origin } & Chromosomal location of CTX prophage (copy no.) & Reference \\
\hline O395 & O1 (classical) & Clinical & Large (1), small (1) & Trucksis et al. $(1998)$ \\
N16961 & O1 (El Tor) & Clinical & Large (1) & Heidelberg et al. $(2000)$ \\
VC44 & O1 (El Tor) & Clinical & Small (2) & Nandi et al. $(2003)$ \\
CO471 & O1 (El Tor) & Clinical & Large (1) & Nandi et al. $(2003)$ \\
VCE232 & O4 & Environmental & Large $(2)^{\star}$, small (2) & This study \\
\hline
\end{tabular}

${ }^{\star}$ CTX prophages are devoid of $\operatorname{ctx} A B$ genes. 
separation of the two chromosomes of $V$. cholerae, undigested washed agarose slices were used directly (Nandi et al., 2003; Trucksis et al., 1998). PFGE was carried out in a Pulsaphor Plus system with a hexagonal electrode array (Amersham Pharmacia Biotech) in $0 \cdot 5 \times$ TAE buffer $(20 \mathrm{mM}$ Tris-acetate, $0.5 \mathrm{mM}$ EDTA, $\mathrm{pH} 8 \cdot 3$ ), essentially as described previously (Nandi et al., 2003). After electrophoresis, gels were stained with ethidium bromide $\left(0.5 \mu \mathrm{g} \mathrm{ml}^{-1}\right)$ and DNA was visualized.

PCR and other molecular genetic techniques. Standard molecular biological methods were followed throughout the study (Sambrook et al., 1989), unless otherwise mentioned. Primers used in PCR experiments are described in Table 2. For hybridization experiments, an $\sim 1.0 \mathrm{~kb}$ fragment of the $c t x A B$ operon or $2.3 \mathrm{~kb}$ of the RS segment were PCR-amplified using the primers CtxAF/CtxBR or Ig1F/RSR (Table 2), respectively, and genomic DNA of the $V$. cholerae El Tor strain N16961 (Table 1) as a template. An $\sim 0.8 \mathrm{~kb}$ Pst $\mathrm{I}-\mathrm{NruI}$ internal fragment of the PCR amplicon obtained by the primer pair OrfU-F/CtxAR (Table 2), using the genomic DNA of the V. cholerae O1 El Tor strain N16961 (Heidelberg et al., 2000; Table 1) as template, was used as a gIII $^{\mathrm{CTX}}$ gene (Heilpern \& Waldor, 2003) probe. Similarly, an $\sim 1.5 \mathrm{~kb} A v a \mathrm{I}-B g l \mathrm{II}$ internal fragment of the $3.7 \mathrm{~kb}$ insert DNA present in the recombinant plasmid pCTCP1 (this study) was used as a probe (called URS probe) for the detection of a new type of RS2-like element identified in this study. The PCR amplification conditions used in this study were as follows: a single step of denaturation of template DNA at $94{ }^{\circ} \mathrm{C}$ for $1 \mathrm{~min}$, followed by amplification of 30 cycles, each of

Table 2. Sequences of primers used in this study

\begin{tabular}{|lll|}
\hline Primer & \multicolumn{1}{c|}{ Primer sequence $\left(\mathbf{5}^{\prime} \mathbf{3}^{\prime} \mathbf{}\right)$} & \multicolumn{1}{c|}{ Use } \\
\hline CtxAF & CTCAGACGGGATTTGTAGGCACG & PCR \\
CtxAR & TCTATCTCTGTAGCCCCTATTACG & PCR \\
CtxBF & GATACACATAATAGAATTAAGGA & PCR, \\
& & sequencing \\
CtxBR & GGTTGCTTCTCATCATCGAACCAC & PCR \\
Ig1F & GAGCCTGTGACACTCACCTTGTAT & PCR \\
RstAR & GCATATGGAACCGACCAAGCAAGAT & PCR \\
RSR & GCTCAGTCAATGCCTTGAGTTG & PCR, \\
& & sequencing \\
RstBF & GTGGTGTGTTCCTTGTTTGAC & PCR \\
OrfUF & GGTGTTATTTGATGGCTGCATG & PCR \\
CepR & AGACCCAGCCACAACTCAAG & PCR, \\
& & sequencing \\
CIIF & CTCACGCTGAACAGCAAGTC & PCR \\
CIIR & TTGCTTGAATCGAAAGGACA & PCR \\
RtxAR & GCTGATAAGCTTCGTCAAGCCAA & PCR \\
TlcF & GATTGTGCGTCTTGCATTTAGG & PCR \\
TlcR & GTGAATAAATCAGGTGTAATGTCG & PCR \\
CtxBF2 & GCCGCAATTAGTATGGCAAA & PCR, \\
& & sequencing \\
CepR2 & CGCCTCGTTCCAGACATAAT & PCR, \\
& & sequencing \\
Ig1aF & GTGAGAACATAAGCGACGTA & Sequencing \\
UR2 & ACCTTCGAGCTTCATATTTCATTC & Sequencing \\
UR3 & CGTAACCGCCATCTGTCATT & Sequencing \\
UR5 & GCTCGTGATTTGGAATTGGT & Sequencing \\
UR6 & GTACCGCTTTAAGGTTGAGCAT & Sequencing \\
& & \\
\hline
\end{tabular}

which consisted of three steps, denaturation at $94{ }^{\circ} \mathrm{C}$ for $30 \mathrm{~s}$, primer annealing at $55-60^{\circ} \mathrm{C}$ (depending upon the primers used) for $30 \mathrm{~s}$, and primer extension at $72{ }^{\circ} \mathrm{C}$ for a time period based on the expected size of the amplicon (usually 1 min per $\mathrm{kb}$ of DNA fragment), followed by a single step of final extension at $72{ }^{\circ} \mathrm{C}$ for $7 \mathrm{~min}$. The PCR assay was carried out in a GeneAmp PCR system (model 9700, Applied Biosystems). The PCR-amplified DNA fragment was always purified by the electroelution method (Sambrook et al., 1989) and was used for various purposes. For labelling of DNA, $\sim 50$ ng DNA was labelled with $\left[\alpha^{32} \mathrm{P}\right] \mathrm{dCTP}$ (Amersham Biosciences) by the random-priming method, using the NEBlot kit (New England Biolabs). For Southern blot hybridization, restrictionenzyme-digested bacterial genomic DNA was separated by electrophoresis, transferred to Hybond-N+ membranes (Amersham Biosciences) and hybridized with a labelled DNA probe at $60{ }^{\circ} \mathrm{C}$ in a hybridization oven (Amersham Biosciences), essentially as described previously (Bhadra et al., 1995; Nandi et al., 2003). The membranes were washed under stringent conditions (Bhadra et al., 1995), dried, and exposed to Kodak X-OMAT AR5 film.

Cloning and DNA sequencing. Initially, a PCR experiment was done using the primers CtxBF ( $c t x B$ gene-specific sequences) and CepR (cep gene-specific sequences) (Table 2) to amplify the $4 \cdot 4 \mathrm{~kb}$ RS2-like element of the small chromosomal CTX prophage of VCE232. Sequencing of the $3^{\prime}$ and $5^{\prime}$ regions of this PCR product using the same primers helped us further in designing two primers, CtxBF2 and CepR2 (Table 2), for PCR amplification of a $\sim 3 \cdot 7 \mathrm{~kb}$ DNA segment that contained the entire RS2-like element (data not shown). A plasmid named pCTCP1 carrying a $3.7 \mathrm{~kb}$ insert was selected for sequencing. Sequencing was done in an automated DNA sequencer (Genetic Analyser 3130, Applied Biosystems) using company-supplied kit reagents and protocols. For initial sequencing, M13 forward and reverse universal primers (New England Biolabs) were used, since the pCR4TOPO cloning vector (Invitrogen) contains M13 forward and reverse primer binding sites. The sequence obtained was subjected to National Center for Biotechnology Information (NCBI) BLASTN version 2.2.10 (http://www.ncbi.nlm. nih.gov/blast/) to check the authenticity and homology of the sequence, followed by the design of several forward and reverse primers (see Table 2) for sequencing the DNA of both strands of the entire $3 \cdot 7 \mathrm{~kb}$ insert.

Nucleotide sequence and other computer-based analysis. DNA sequence data were compiled and analysed by the DNASIS program (Hitachi). BLASTN version 2.2.10 was used to search for homologous sequences in the database. The NCBI ORF finder tool (http:// www.ncbi.nlm.nih.gov/gorf/gorf.html) was used to identify potential ORFs within the sequenced fragment. The ORFs were subsequently subjected to a database search using BLASTP version 2.2.10 (http:// www.ncbi.nlm.nih.gov/blast/Blast.cgi).

\section{RESULTS AND DISCUSSION}

\section{Two copies of the CTX prophage in tandem are located in the small chromosome of VCE232}

We have reported recently that the non-O1, non-O139 strain VCE232 harbours the $\operatorname{ctx} A B$ genes in its small chromosome (Bhattacharya et al., 2006). In this study, fine mapping with the restriction enzymes AvaI, BglI, BglII, PstI and $X b a \mathrm{I}$, which have single sites either in the RS2 or in the core of the phage genome (Bhadra et al., 1995; Mekalanos, 1983; Nandi et al., 2003; Pearson et al., 1993), and using the $\operatorname{ctx} A B$ region as a probe in hybridization experiments, helped us in deducing the genetic organization of the CTX 
prophage in the small chromosome of VCE232. When the $c t x A B$ probe was used, two fragments of equal intensity were observed in the autoradiogram, indicating the presence of two copies of the CTX prophage in VCE232 (Fig. 2a, b). Further detailed analyses of the autoradiograms and a comparison of the data with the similar information available for the $V$. cholerae O1 biotype El Tor strain VC44 (Table 1) reported previously (Nandi et al., 2003) indicated that the small chromosomal CTX prophages are arranged in tandem in strain VCE232, and that the two cores of the phages are connected by an unusual $3.5 \mathrm{~kb}$ DNA segment, instead of the canonical $2 \cdot 4 \mathrm{~kb}$ RS2 element found in epidemic V. cholerae strains (Waldor \& Mekalanos, 1996). Our analysis indicates that the genome size of the CTX prophage present in VCE232 should be $\sim 8.0 \mathrm{~kb}(3.5 \mathrm{~kb}$ of RS2-like element $+4.5 \mathrm{~kb}$ of core $=8.0 \mathrm{~kb}$ ), instead of the $6.9 \mathrm{~kb}(2.4 \mathrm{~kb}$ of RS2 $+4.5 \mathrm{~kb}$ of core $=6.9 \mathrm{~kb})$ found in epidemic V. cholerae strains (Mekalanos, 1983; Pearson et al., 1993; Waldor \& Mekalanos, 1996; Waldor et al., 1997). Hybridization of PFGE-separated NotI fragments of VCE232 with the $\operatorname{ctx} A B$ probe produced only one signal in the $130 \mathrm{~kb}$ region (data not shown), which is a similar result to that of $V$. cholerae El Tor strains containing tandemly arranged CTX prophages in the small chromosome (Nandi et al., 2003). All this evidence indicated that the two copies of the CTX prophage in the VCE232 genome are likely to be arranged in tandem. It also appears that the CTX prophage identified in VCE232 has a novel structure not reported before.

\section{Presence of two tandemly arrayed copies of pre-CTX prophages in the large chromosome of VCE232}

Apart from the $\operatorname{ctx} A B$ probe, we also tested another core gene, $g I I I^{\text {CTX }}$ (Fig. 1), in our hybridization experiments, for two reasons: first, it has been reported that $V$. cholerae may possess CTX prophages devoid of $c t x A B$ genes, called preCTX prophages (Boyd et al., 2000; Jiang et al., 2003; Li et al., 2003); second, we wished to confirm further the presence of the CTX prophage in the small chromosome of VCE232. PFGE of intact chromosomal DNAs of VCE232 along with control V. cholerae strains, namely, O395, N16961 and VC44 (Table 1), was performed to separate their two chromosomes (Fig. 3a) followed by transfer to a membrane and hybridization first with the $\operatorname{ct} x A B$ probe (Fig. $3 \mathrm{~b}$ ) and, after stripping, with the $g I I I^{\mathrm{CTX}}$ probe (Fig. 3c). While the $\operatorname{ctx} A B$ probe hybridized with the small chromosome of VCE232, surprisingly, the $g I I I^{C T X}$ gene hybridized with both the chromosomes of VCE232 (Fig. 3c). The O1 strains used as controls, however, gave identical results with both $c t x A B$ and $g I I I^{C T X}$ probes (Fig. 3b, c). Furthermore, the (a)

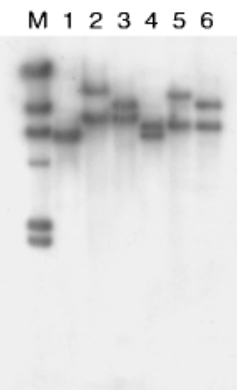

(b)

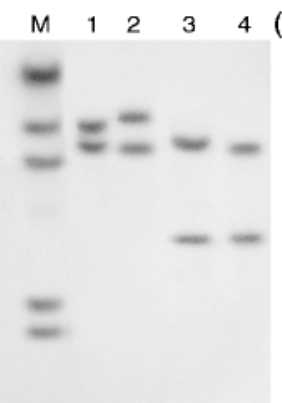

(d)

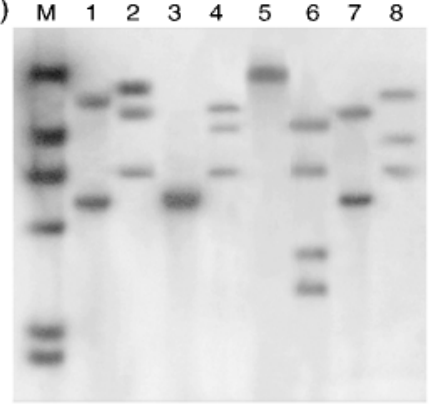

(c) $M \begin{array}{lllllllllll}M & 1 & 2 & 3 & 4 & 5 & 6 & 7 & 8 & 9 & 10\end{array}$

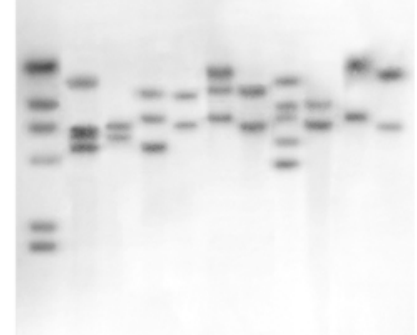

(e) M $\begin{array}{llll}1 & 2 & 3 & 4\end{array}$

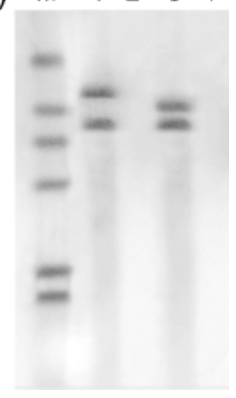

Fig. 2. Southern hybridizations of enzymedigested $V$. cholerae genomic DNA with $\operatorname{ctx} A B(\mathrm{a}, \mathrm{b}), g^{\prime / I^{\mathrm{CTX}}}$ (c) RS (d) and URS (e) as probes. (a) Lanes: 1, 2 and 3, Aval, Bg/ll and Pstl digests of VCE232, respectively; 4, 5 and 6, Aval, Bg/ll and Pstl digests of VC44, respectively. (b) Xbal-digested genomic DNA of VCE232 (lane 1) and VC44 (lane 2); Bgll-digested genomic DNA of VCE232 (lane 3) and VC44 (lane 4). (c) Aval digests of VCE232 (lane 1) and VC44 (lane 2); Bgll digests of VCE232 (lane 3) and VC44 (lane 4); Bg/ll digests of VCE232 (lane 5) and VC44 (lane 6); Pstl digests of VCE232 (lane 7) and VC44 (lane 8); Xbal digests of VCE232 (lane 9) and VC44 (lane 10). The $5.5 \mathrm{~kb}$ band corresponds to the restriction fragment originating from the preCTX prophages present in tandem in the large chromosome of VCE232. (d) Lanes 1-8 as explained in (c). Intensity of the single $\mathrm{Bg} / \mathrm{l}$ band (lane 3 ) detected by the RS probe indicates the presence of multiple co-migrating fragments (see Fig. 6). (e) Bg/ll digests of VCE232 (lane 1) and VC44 (lane 2); Pstl digests of VCE232 (lane 3) and VC44 (lane 4). 'M' denotes $\lambda$ DNA digested with Hindlll used as a molecular mass marker (from top to bottom the fragment sizes are $23 \cdot 1,9 \cdot 4,6 \cdot 5,4 \cdot 3,2 \cdot 3$ and $2 \cdot 0 \mathrm{~kb})$. 


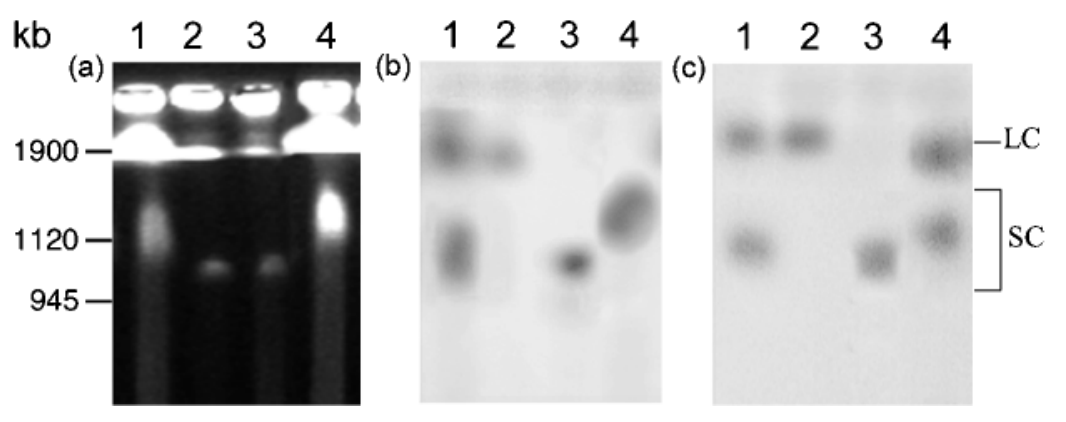

Fig. 3. Chromosomal location of the pre-CTX prophage in $V$. cholerae strain VCE232. (a) Ethidium bromide-stained PFGE profile of undigested intact genomic DNAs of $V$. cholerae strains. (b) Southern hybridization of DNAs shown in panel (a) probed with the ct $x A B$ genes. (c) The blot shown in panel (b) was stripped and reprobed with the g/l/CTX gene. Lanes: 1, O395; 2, N16961; 3, VC44; 4, VCE232. LC and SC indicate large and small chromosomes of $V$. cholerae, respectively. Numbers in the left-hand margin indicate yeast chromosomes as molecular mass markers.

NotI-digested PFGE-separated genome of VCE232 also hybridized with the $g I I I^{\text {CTX }}$ probe, apart from a $130 \mathrm{~kb}$ band originating from the small chromosome (the same band was detectable by the $\operatorname{ct} x A B$ probe), an additional Not I fragment of $\sim 97 \mathrm{~kb}$ was observed in the autoradiogram (data not shown). The detection of signals in both the chromosomes of VCE232 with the $g I I I^{\mathrm{CTX}}$ probe (Fig. 3c), but only in the small chromosome with the $c t x A B$ probe (Fig. 3b), indicated the probable presence of a pre-CTX prophage in the large chromosome. This observation prompted us to determine the genetic organization of the pre-CTX prophage in VCE232. Extensive Southern hybridization analyses of AvaI-, BglI-, BglII-, PstI- and XbaI-digested genomic DNA of VCE232 using the $g I I I^{\mathrm{CTX}}$ probe (Fig. 2c), and comparison of the data with that of the similarly digested, but probed with the $\operatorname{ct} x A B$ genes, genomic DNA of VCE232, as described in the previous section, indicated that the large chromosome of VCE232 also harbours two copies of the pre-CTX prophage arranged in tandem (see below). Interestingly, unlike the RS2 element of the small chromosomal CTX prophage, the RS2 segment of the preCTX prophage of the large chromosome appears to be similar to that of RS2 of $V$. cholerae O1 strains, which is supported by the fact that an RS2 probe generated from the $V$. cholerae O1 El Tor strain N16961 hybridized only with those restriction fragments which originated from the preCTX prophage region of the large chromosome (Fig. 2d). To our knowledge, this type of structural organization of pre-CTX and CTX prophages in $V$. cholerae per se has not been reported previously, and the information obtained from this study raises the possibility that non-O1, non-O139 $V$. cholerae may play a role in the evolution of diverse CTX prophages, which in turn may influence the emergence of new pathogenic clones of $V$. cholerae.

\section{Site of integration of the pre-CTX and CTX prophages in VCE232}

In the large chromosome of epidemic strains, the CTX prophage is integrated between the 'toxin-linked cryptic element' or tlc and the 'repeats in toxin' or $r t x$ loci (Davis et al., 2000; Heidelberg et al., 2000; Lin et al., 1999; Rubin et al., 1998). In the small chromosome of $V$. cholerae classical strains (Davis et al., 2000) and in certain clinical V. cholerae El Tor strains (Nandi et al., 2003), it is integrated between the traF and $y c i H$ genetic loci (Davis et al., 2000). In both cases the integration site $(a t t B)$ is a 17 bp sequence, called the end repeat, which is almost identical to an 18 bp repeat sequence present in the phage genome (Davis \& Waldor, 2000). To determine the integration sites of pre-CTX and CTX prophages in the large and small chromosomes of VCE232, respectively, site-specific PCR assays were performed. As controls, strains N16961 (CTX prophage present in the large chromosome) and VC44 (CTX prophage present in the small chromosome) belonging to the $V$. cholerae El Tor biotype were employed. Two primers were designed, called CIIF (forward) and CIIR (reverse) (Table 2), using the small chromosomal sequences (Heidelberg et al., 2000) which flank the predicted CTX prophage integration site, i.e. the traF and $y c i H$ gene regions. Using the primers CtxAF and CIIR (Table 2), and VCE232 or VC44 genomic DNA as template, an amplicon of $\sim 1.4 \mathrm{~kb}$ was obtained and, as expected, no amplicon was obtained in the case of N16961 (Fig. 4). The result strongly suggests that the CTX prophage is integrated in the same locus of the small chromosome of VCE232 as in epidemic $V$. cholerae strains (Nandi et al., 2003). Sequencing of the $1.4 \mathrm{~kb}$ PCR product obtained from strains VCE232 and VC44 confirmed this conclusion (data not shown). Furthermore, while the $V$. cholerae El Tor strains N16961 and CO471 (Table 1), which carry the CTX prophage in the large chromosome only (Heidelberg et al., 2000; Nandi et al., 2003), gave the expected PCR amplicon of $0.8 \mathrm{~kb}$ with primers CIIF and CIIR due to the absence of the CTX prophage between the $t r a F$ and $y c i H$ genes, called the 'empty site' (Davis et al., 2000), V. cholerae strains VCE232 and VC44 (as control strains) both failed to give any PCR product using the same primer pair (Fig. 4). The results also support the integration of the CTX prophage in the traF$y c i H$ region of the small chromosome of VCE232, as in epidemic strains. Similarly, to determine whether the preCTX prophage in the large chromosome of VCE232 integrates between the $t l c$ and $r t x$ loci, OrfUF and RtxAR primers (Table 2) were used for PCR amplification. While in the $V$. cholerae El Tor strain CO471, which has an intact 


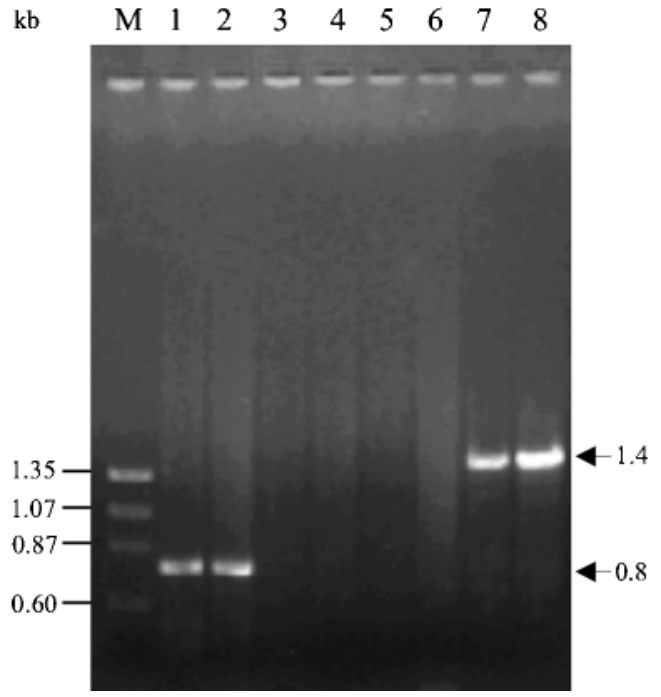

Fig. 4. Site of integration of the CTX prophage in the small chromosome of VCE232. PCR amplification was done with CIIF/CIIR primers (lanes 1-4) and CtxAF/CIIR primers (lanes 5-8) using genomic DNA of $V$. cholerae as template. Lanes: 1 and $5, \mathrm{~N} 16961 ; 2$ and $6, \mathrm{CO} 471 ; 3$ and $7, \mathrm{VC} 44 ; 4$ and 8 , VCE232. Numbers in the right-hand margin indicate the sizes of the PCR amplicons (kb). M, $\phi$ X174 DNA digested with Haelll and used as molecular size markers.

core and no downstream RS1 element (Nandi et al., 2003), an expected amplicon size of $\sim 6.6 \mathrm{~kb}$ was obtained, the VCE232 genomic DNA when used as template also yielded an amplicon, but its size was $1 \cdot 1 \mathrm{~kb}$ less than that of CO471 (data not shown), which was due to the presence of tandemly arranged pre-CTX prophages in the same locus, but devoid of $\operatorname{ctx} A B$ genes. On the other hand, both by hybridization and PCR analysis using primers TlcF and TlcR
(Table 2), it was confirmed that strain VCE232 lacks the tlc element (data not shown). This is not surprising, because it has been reported elsewhere that in non-O1, non-O139 strains the tlc element is absent (Dalsgaard et al., 2001). The $5^{\prime}$ region of the pre-CTX prophage integration in the large chromosome of VCE232 is still being determined.

\section{Cloning and sequence analyses of the unique RS2-like element of VCE232}

The RS2-like element was cloned as a $3.7 \mathrm{~kb}$ fragment in plasmid pCTCP1 and sequenced. Analysis revealed three complete ORFs within the $3.7 \mathrm{~kb}$ region (Fig. 5), none of which at the nucleotide level strongly matched the genes of the RS element of CTX prophages reported for $V$. cholerae. However, there was no doubt that the RS2-like sequence of VCE232 was of bacteriophage origin. A 114 amino acidcoding ORF (nucleotide positions 704-1048), lying immediately adjacent to ig1 (nucleotide positions 149-703), showed about $36 \%$ identity at the amino acid level with the $\mathrm{RstR}^{\text {class }}$ of the RS2 region present in V. cholerae strain 569B (accession no. AAC24223; Kimsey \& Waldor, 1998). Further analysis of this ORF also revealed homology with helixturn-helix DNA-binding proteins, a characteristic feature of many bacteriophage repressors (Aggarwal et al., 1988), including Rst ${ }^{\mathrm{ET}}, \mathrm{Rst}^{\text {class }}$ and Rst $\mathrm{R}^{\mathrm{Calc}}$ (Davis et al., 1999; Kimsey \& Waldor, 2004; Waldor et al., 1997). Based on its position, orientation and identity to the RstR of the classical CTX phage, this particular ORF may encode an RstR-like repressor protein, and thus the ORF may be provisionally designated rstR-232 according to Mukhopadhyay et al. (2001). Adjacent to $r s t R-232$, a 709 amino acid-coding ORF (nucleotide positions 1172-3301) was observed. The product of this ORF showed $49 \%$ identity at the amino acid level with the 716 amino acid replication proteincoding ORF of the fs- 2 phage of $V$. cholerae O139 strain

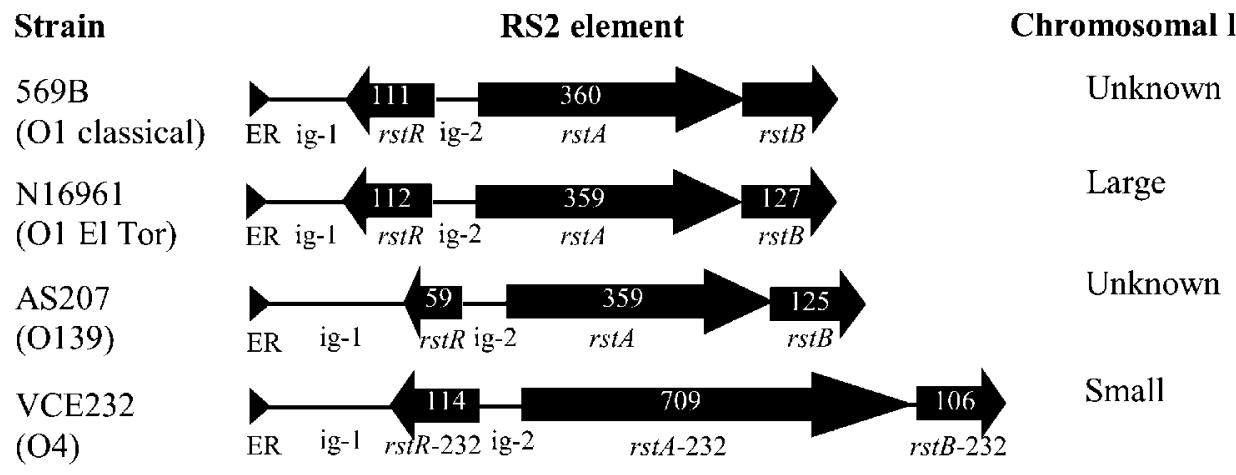

Fig. 5. Comparison of the genetic structures of different RS2 elements found in $V$. cholerae. The triangle represents an end repeat (ER). Arrows indicate genes present in the RS2 element with their direction of transcription, and the number within each arrow indicates the number of amino acids encoded by a gene, except for the $r s t B$ gene of the classical strain $569 \mathrm{~B}$, for which only a partial sequence is available. The thin lines indicate intergenic DNA segments. Parentheses indicate the serotype/biotype of $V$. cholerae. GenBank accession numbers of each of the RS2 elements are as follows: 569B, AF055890 (Kimsey \& Waldor, 1998); N16961, AE004224 (Heidelberg et al., 2000); AS207, AF110029 (Davis et al., 1999); VCE232, DQ288668 (this study). 


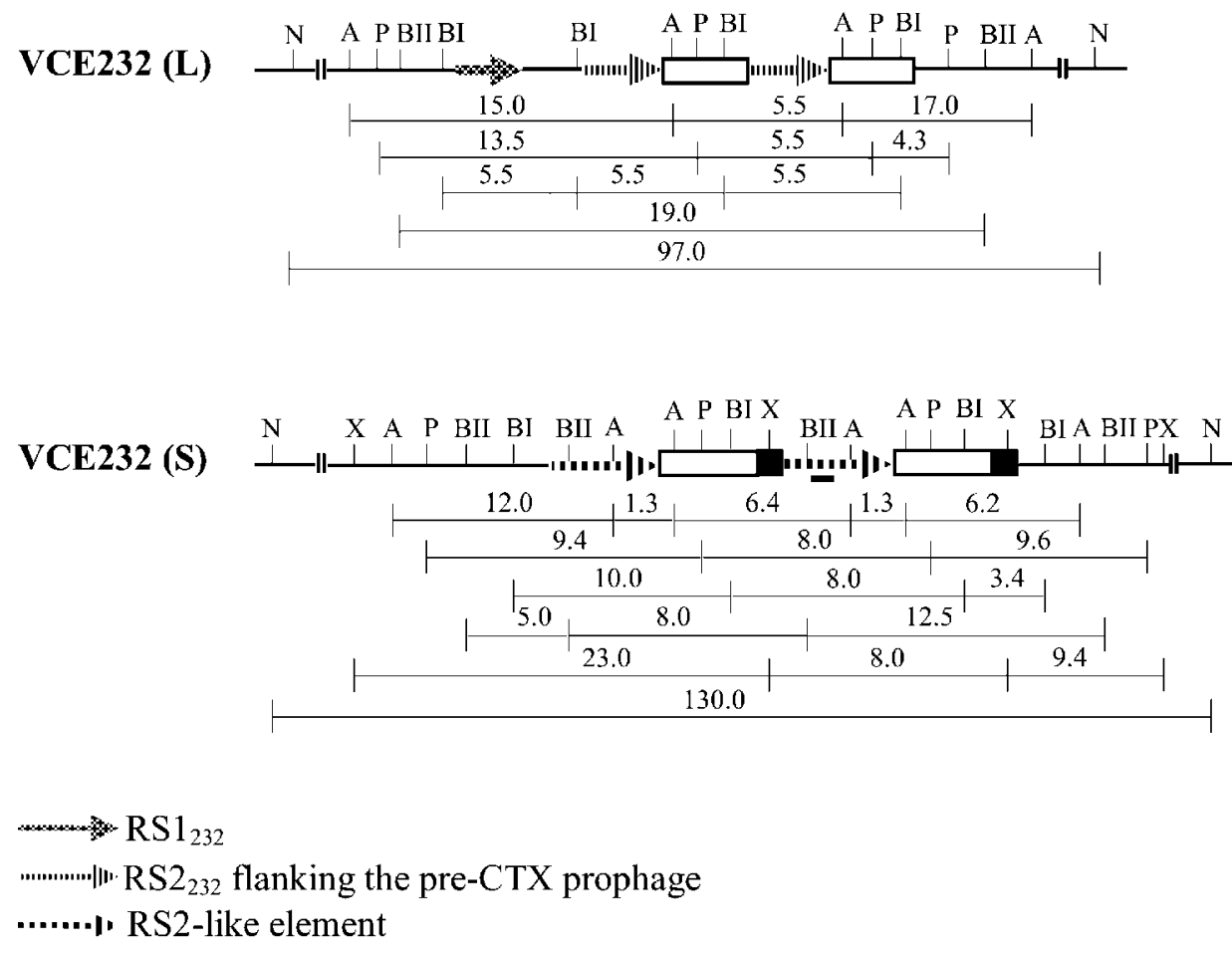

Fig. 6. Schematic representation of the genetic organization of pre-CTX and CTX prophages in the large (L) and small (S) chromosomes of strain VCE232, respectively. Restriction maps (not drawn to scale) of the genomic regions are also shown. Various restriction fragment sizes (in $\mathrm{kb}$ ) obtained by hybridization experiments (see Fig. 2) are indicated below each representation of the genetic organization of the prophages. Thin lines represent chromosomal DNA. A rectangular box represents a truncated core devoid of $c t x A B$ genes, a filled box indicates $c t x A B$ genes, and a solid bar indicates the location of the URS probe. Diverse RS elements present in strain VCE232 are shown. A, Aval; BI, Bgll; Bll, Bglll; N, Notl; P, Pstl; X, $X$ bal.

MDO14 (accession no. BAA33476) reported by Ikema \& Honma (1998). This ORF was followed by a 106 amino acid-coding ORF (nucleotide positions 3350-3670) that showed $39 \%$ identity at the amino acid level with the product of orf104 (accession no. BAA33477) of an fs-2 phage, which corresponds to gene $V$ (single-stranded DNAbinding protein of coliphage) involved in DNA replication (Ikema \& Honma, 1998; Mukhopadhyay et al., 2001). These two ORFs were provisionally designated $r s t A-232$ and $r s t B$ 232 , respectively, based on their positions, arrangement and direction of transcription in the RS element, and the similarity of their products with that of fs-2 phage replication proteins. The overall similarity in genetic organization, including location and homology, presence of intergenic regions and direction of transcription of the genes, of the RS2-like element of VCE232 with that of a filamentous single-stranded phage such as fs- 2 of $V$. cholerae O139, as well as with the RS2 element of the $V$. cholerae classical strain 569B, suggests that the small chromosomal RS2-like element of VCE232 is most likely a part of the filamentous CTXФ integrated in its genome. A schematic representation of this newly identified $3.5 \mathrm{~kb}$ RS2-like element (nucleotide positions 131-3670) of VCE232 and its comparison with the common RS2 elements found in some of the epidemic $V$. cholerae strains is shown in Fig. 5. Thus, the organization of the VC232 RS2-like element is similar to that of the other $V$. cholerae strains.

\section{Organization of CTX prophages in the genome of $V$. cholerae strain VCE232}

Hybridization with a canonical RS probe (Fig. 2d) and with a $1.5 \mathrm{~kb}$ internal fragment of the unique RS2-like element of VCE232 obtained by AvaI and BglII double digestion of the $3.7 \mathrm{~kb}$ insert DNA of plasmid pCTCP1, known as the URS probe (Fig. 2e), allowed the positions to be determined of two different types of RS2 elements in the large and small chromosomes of VCE232 (Fig. 6). In addition to hybridization experiments, region-specific PCR assays also helped to determine the organization of the CTX genetic elements in VCE232. The presence of an RS1 element in the VCE232 genome was confirmed by amplifying its genomic DNA with the primers Ig1F and RSR (Table 2), for which the $V$. cholerae El Tor strain VC44 served as a control (Nandi et al., 2003). PCR of VCE232 genomic DNA using either the primer pair CtxAF (ctxA-specific forward primer) and RSR ( $r s t C$-specific reverse primer) or OrfUF ( $g I I I^{\mathrm{CTX}}$-specific forward primer) and RSR gave no amplicon (data not 
shown), indicating the absence of an RS1 downstream of the prophages present in the large or small chromosome. Similarly, PCR assay using the VCE232 genomic DNA as template and the primers CIIF (Table 2) and RSR (rstCspecific reverse primer) also failed to give any amplicon (data not shown), suggesting the absence of an RS1 element immediately upstream of the CTX prophage in the small chromosome of VCE232. To determine the upstream position of the RS1 of the large chromosome, a PCR experiment was done using primers RstBF and RstAR (Table 2). If RS1 is located immediately upstream of RS2 in VCE232, then, according to the whole-genome sequence data (Heidelberg et al., 2000), one would expect an amplicon size of $\sim 1.5 \mathrm{~kb}$ using primers RstBF and RstAR. Interestingly, while the control $V$. cholerae strain N16961 (Heidelberg et al., 2000) gave an expected PCR amplicon of $1.5 \mathrm{~kb}$, in the case of VCE232 the same primer pair gave a $2.0 \mathrm{~kb}$ amplicon (data not shown). It seems that the upstream RS1 is not arranged exactly in tandem; rather, it is very close to the pre-CTX prophage, and thus it may be considered as a free RS1 element, as shown schematically in Fig. 6.

In conclusion, the results of this study have shown that a non-O1, non-O139 V. cholerae strain can be simultaneously infected by diverse СТХ $\Phi$, and that the genetic organization of its genome is highly complex. Further studies on the organization and function of the CTX prophages of environmental toxigenic strains of $V$. cholerae would enhance our understanding of how these environmental strains play a role in the emergence of new variants of toxigenic $V$. cholerae.

\section{ACKNOWLEDGEMENTS}

We are indebted to Professor Siddhartha Roy for his constant support to this study. We are grateful to Dr T. Ramamurthy, National Institute of Cholera and Enteric Diseases, for generous donation of $V$. cholerae strains. The work was supported by research grant SMM003 from the Council of Scientific and Industrial Research (CSIR), Government of India. D. M. is grateful to the CSIR for a Senior Research Fellowship.

\section{REFERENCES}

Aggarwal, A. K., Rodgers, D. W., Drottar, M., Ptashne, M. \& Harrison, S. C. (1988). Recognition of a DNA operator by the repressor of phage 434: a view at high resolution. Science 242, 899-907.

Bhadra, R. K., Roychoudhury, S., Banerjee, R. K., Kar, S., Majumdar, R., Sengupta, S., Chatterjee, S., Khetawat, G. \& Das, J. (1995). Cholera toxin (CTX) genetic element in Vibrio cholerae O139. Microbiology 141, 1977-1983.

Bhattacharya, T., Chatterjee, S., Maiti, D., Bhadra, R. K., Takeda, Y., Nair, G. B. \& Nandy, R. K. (2006). Molecular analysis of the $r s t R$ and orfU genes of the CTX prophages integrated in the small chromosomes of environmental Vibrio cholerae non-O1, non-O139 strains. Environ Microbiol 8, 526-534.

Bik, E. M., Bunschoten, A. E., Gouw, R. D. \& Mooi, F. R. (1995). Genesis of the novel epidemic Vibrio cholerae O139 strain: evidence for horizontal transfer of genes involved in polysaccharide synthesis. EMBO J 14, 209-216.

Boyd, E. F., Heilpern, A. J. \& Waldor, M. K. (2000). Molecular analyses of a putative СТX $\Phi$ precursor and evidence for independent acquisition of distinct СТХ $\Phi$ s by toxigenic Vibrio cholerae. J Bacteriol 182, 5530-5538.

Dalsgaard, A., Serichantalergs, O., Forslund, A., Lin, W., Mekalanos, J., Mintz, E., Shimada, T. \& Wells, J. G. (2001). Clinical and environmental isolates of Vibrio cholerae serogroup O141 carry the CTX phage and the genes encoding the toxin-coregulated pili. J Clin Microbiol 39, 4086-4092.

Davis, B. M. \& Waldor, M. K. (2000). СТХ $\Phi$ contains a hybrid genome derived from tandemly integrated elements. Proc Natl Acad Sci U S A 97, 8572-8577.

Davis, B. M., Kimsey, H. H., Chang, W. \& Waldor, M. K. (1999). The Vibrio cholerae $\mathrm{O} 139$ Calcutta bacteriophage CTX $\Phi$ is infectious and encodes a novel repressor. J Bacteriol 181, 6779-6787.

Davis, B. M., Moyer, K. E., Boyd, E. F. \& Waldor, M. K. (2000). CTX prophages in classical biotype Vibrio cholerae: functional phage genes but dysfunctional phage genomes. J Bacteriol 182, 6992-6998.

Davis, B. M., Kimsey, H. H., Kane, A. V. \& Waldor, M. K. (2002). A satellite phage-encoded antirepressor induces repressor aggregation and cholera toxin gene transfer. EMBO J 21, 4240-4249.

Heidelberg, J. F., Eisen, J. A., Nelson, W. C. \& 29 other authors (2000). DNA sequence of both chromosomes of the cholera pathogen Vibrio cholerae. Nature 406, 477-483.

Heilpern, A. J. \& Waldor, M. K. (2003). pIII ${ }^{\mathrm{CTX}}$, a predicted CTXФ minor coat protein, can expand the host range of coliphage fd to include Vibrio cholerae. J Bacteriol 185, 1037-1044.

Ikema, M. \& Honma, Y. (1998). A novel filamentous phage, fs-2, of Vibrio cholerae O139. Microbiology 144, 1901-1906.

Jiang, S., Chu, W. \& Fu, W. (2003). Prevalence of cholera toxin genes (ctxA and zot) among non-O1/O139 Vibrio cholerae strains from Newport Bay, California. Appl Environ Microbiol 69, 7541-7544.

Kaper, J. B., Morris, J. G., Jr \& Levine, M. M. (1995). Cholera. Clin Microbiol Rev 8, 48-86.

Kimsey, H. H. \& Waldor, M. K. (1998). СТХФ immunity: application in the development of cholera vaccines. Proc Natl Acad Sci U S A 95, 7035-7039.

Kimsey, H. H. \& Waldor, M. K. (2004). The CТХФ repressor RstR binds DNA cooperatively to form tetrameric repressor-operator complexes. J Biol Chem 279, 2640-2647.

Li, M., Shimada, T., Morris, J. G., Jr, Sulakvelidze, A. \& Sozhamannan, S. (2002). Evidence for the emergence of non-O1 and non-O139 Vibrio cholerae strains with pathogenic potential by exchange of $\mathrm{O}$-antigen biosynthesis regions. Infect Immun 70, 2441-2453.

Li, M., Kotetishvili, M., Chen, Y. \& Sozhamannan, S. (2003). Comparative genomic analyses of the Vibrio pathogenicity island and cholera toxin prophage regions in nonepidemic serogroup strains of Vibrio cholerae. Appl Environ Microbiol 69, 1728-1738.

Lin, W., Fullner, K. J., Clayton, R., Sexton, J. A., Rogers, M. B., Calia, K. E., Calderwood, S. B., Fraser, C. \& Mekalanos, J. J. (1999). Identification of a Vibrio cholerae RTX toxin gene cluster that is tightly linked to the cholera toxin prophage. Proc Natl Acad Sci U S A 96, 1071-1076.

Mekalanos, J. J. (1983). Duplication and amplification of toxin genes in Vibrio cholerae. Cell 35, 253-263.

Mukhopadhyay, A. K., Chakraborty, S., Takeda, Y., Nair, G. B. \& Berg, D. E. (2001). Characterization of VPI pathogenicity island and СТХ $\Phi$ prophage in environmental strains of Vibrio cholerae. J Bacteriol 183, 4737-4746. 
Nair, G. B., Sarkar, B. L., De, S. P., Chakraborti, M. K., Bhadra, R. K. \& Pal, S. C. (1988). Ecology of Vibrio cholerae in the fresh water environs in Calcutta, India. Microb Ecol 15, 203-215.

Nandi, S., Maiti, D., Saha, A. \& Bhadra, R. K. (2003). Genesis of variants of Vibrio cholerae O1 biotype El Tor: role of CTXФ array and its position in the genome. Microbiology 149, 89-97.

Pearson, G. D., Woods, A., Chiang, S. L. \& Mekalanos, J. J. (1993). CTX genetic element encodes a site-specific recombination system and an intestinal colonization factor. Proc Natl Acad Sci U S A 90, 3750-3754.

Ramamurthy, T., Garg, S., Sharma, R. \& 8 other authors (1993). Emergence of novel strain of Vibrio cholerae with epidemic potential in southern and eastern India. Lancet 341, 703-704.

Rubin, E. J., Lin, W., Mekalanos, J. J. \& Waldor, M. K. (1998). Replication and integration of Vibrio cholerae cryptic plasmid linked to the CTX prophage. Mol Microbiol 28, 1247-1254.

Saha, A., Haralalka, S. \& Bhadra, R. K. (2004). A naturally occurring point mutation in the 13 -mer $\mathrm{R}$ repeat affects the oriC function of the large chromosome of Vibrio cholerae O1 classical biotype. Arch Microbiol 182, 421-427.

Sambrook, J., Fritsch, E. F. \& Maniatis, T. (1989). Molecular Cloning: a Laboratory Manual, 2nd edn. Cold Spring Harbor, NY: Cold Spring Harbor Laboratory.

Trucksis, M., Michalski, J., Deng, Y. K. \& Kaper, J. B. (1998). The Vibrio cholerae genome contains two unique circular chromosomes. Proc Natl Acad Sci U S A 95, 14464-14469.

Waldor, M. K. \& Mekalanos, J. J. (1994). Emergence of a new cholera pandemic: molecular analysis of virulence determinants in Vibrio cholerae $\mathrm{O} 139$ and development of a live vaccine prototype. J Infect Dis 170, 278-283.

Waldor, M. K. \& Mekalanos, J. J. (1996). Lysogenic conversion by a filamentous phage encoding cholera toxin. Science 272, 1910-1914.

Waldor, M. K., Rubin, E. J., Pearson, G. D., Kimsey, H. \& Mekalanos, J. J. (1997). Regulation, replication, and integration functions of the Vibrio cholerae CTXФ are encoded by region RS2. Mol Microbiol 24, 917-926. 